\title{
$77 \mathrm{~K}$ 下 $\mathrm{Bi}_{2} \mathrm{Sr}_{2} \mathrm{CaCu}_{2} \mathrm{O}_{8}$ 单晶 $\mathrm{Bi}-\mathrm{O}$ 面的 高分辨的 STM 图象
}

\author{
陶宏杰 尹潮杨乾声赵忠贤 \\ (中国科学院物理研究所超导国家重点实验室,北宗100080)
}

高凡 戴长春 白春礼

(中国科学院化学研究所, 北京100080)

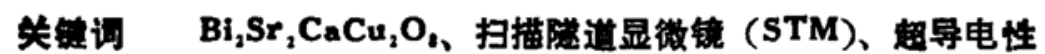

扫描隧道显微镜(STM)是一种新型的、不断发展着的表面分析仪器, 能够提供材料表 面 的原子结构和电子性质等重要信息. 用 STM 研究高温超导体已受到广泛的关注; 其中研究 得最多的是 $\mathrm{Bi}_{2} \mathrm{Sr}_{2} \mathrm{CaCu}_{2} \mathrm{O}_{8}$ (简记 $\mathrm{Bi}-2212$ ) 单晶. 因为通过解理 $\mathrm{Bi}-2212$ 单晶能得到光洁 的、在空气中和在真空下都很稳定的 $\mathrm{Bi}-\mathrm{O}$ 面, 便于 STM 研究. 此外, 现在已经一致认识到 铜氧化物高温超导体的超导电性主要来源于 $\mathrm{Cu}-\mathrm{O}$ 层. 不过, 以 $\mathrm{Bi}-2212$ 为例, $\mathrm{Bi}-\mathrm{O}$ 层也 起着向 $\mathrm{Cu}-\mathrm{O}$ 层提供空穴型载流子, 将部分 $\mathrm{Cu}^{2+}$ 离子变成 $\mathrm{Cu}^{+3}$ 离子, 使 $\mathrm{Bi}-2212$ 变成导 体或超导体的作用 ${ }^{[1}$. 因此, 深人研究 $\mathrm{Bi}-\mathrm{O}$ 层的结构和电子性质, 对理解 $\mathrm{Bi}-2212$ 的超导 电性的来源是很有意义的.

关于 Bi-2212 单晶的结构性质, STM 研究已提供了有价值的信息, 但也还遗留着尚未 解决的问题. Kirk 等人证在室温下用 STM 对 Bi-2212 单晶的 Bi-O 面进行观察, 看到了 平均周期为 $27.2 \AA$ 的一维的无公度调制结构, 即沿 $a$ 方向发展的结构调制. 这种一维调制结 构也被其他研究组用 STM 观察到 ${ }^{[2-10]}$. 关于这个用 STM 观察到的一维无公度调制结构的 形成机制, Kirk 等人隄出了成列的 $\mathrm{Bi}$ 原子丢失模型, 即沿〈110 方向每隔 $9.6 \pm 0.2$ 个 Bi 位, 即有一个 Bi 原子丢失, 从而引起表面晶格变形, 形成此调制结构. 然而 Shih 等人 ${ }^{[3]}$ 和以 后的研究者们声称没有观察到成列的 $\mathrm{Bi}$ 原子丢失, 所观察到的是有成列的 $\mathrm{Bi}$ 原子被压下去 了, 从而形成此调制结构. 此外, Shih 等人还观察到 Bi 原子链在 $a b$ 面内有水平方向的正 弦形调制结构. 迄今对 Bi-2212 的 STM 研究, 多数是在室温下进行的, 低温下原子分辨的 工作还比较少,且图象清晰度也不够高 ${ }^{[8-10]}$.

本文报道了用我们自己研制的低温扫描隧道显微镜(LT-STM)在液氮温度下 $(77 \mathrm{~K})$, 对 $\mathrm{Bi}-2212$ 单晶 $\mathrm{Bi}-\mathrm{O}$ 面所作的原子分辨的观察和所获得的迄今最高分辨的原子图象. 我们 的结果的一个令人注目的特点是 $\mathrm{Bi}-2212$ 单晶的 $\mathrm{Bi}-\mathrm{O}$ 面在水平面内 ( $a b$ 面内) 是完整的 四方格子, 不存在水平方向的位置调制, 也没有显示出成列的 $\mathrm{Bi}$ 原子丢失或被压下去, 因此, 没有连贯的一维超结构, 所观察到的只有在 $a b$ 面上下 ( $c$ 方向) $\mathrm{Bi}$ 原子位置的起伏.

1993-05-12收稿. 


\section{1 实 验方法}

Bi-2212 单晶是用分析纯的 $\mathrm{Bi}_{2} \mathrm{O}_{3}, \mathrm{SrCO}_{3}, \mathrm{CaCO}_{3}$ 和 $\mathrm{CuO}$ 粉末, 以元素比 $\mathrm{Bi}: \mathrm{Sr}: \mathrm{Ca}$ : $\mathbf{C u}-2.4: 2: 1: 2$ 配制, 经精细混合和研磨, 用定向结晶方法生长的. 本实验所用的单晶的尺 寸为 $5 \mathrm{~mm} \times 5 \mathrm{~mm} \times 10 \mu \mathrm{m}$. X 射线行射测定为 2212 单相,实验观测面为 $(001)$ 面. 晶体的 如导转变温度为 $85 \mathrm{~K}$.

关于 $\mathrm{Bi}-2212$ 单晶, 已经用 X射线定出其晶胞参数: $a-b=5.4 \AA, c-30.9 \AA$, 属

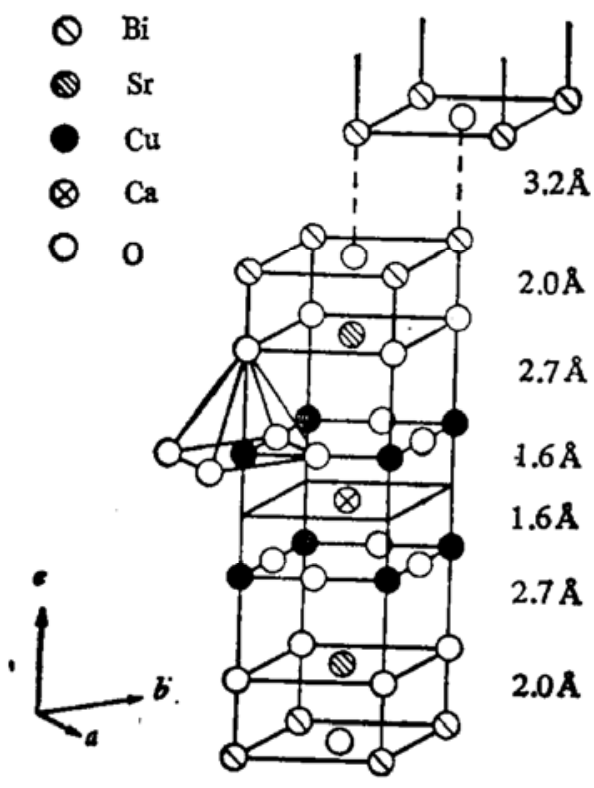

图1 Bi-2212 的空间结构图及各原子层间距离 正交晶系. 图 1 给出 $\mathrm{Bi}-2212$ 单晶 $1 / 2$ 元胞的 空间结构及沿 $c$ 方向各原子层之间的距离. 由 于相邻 $\mathrm{Bi}-\mathrm{O}$ 层之间的间距为 $3.2 \AA$, 二层之 间的耦合很弱, 容易沿此界面进行解理. 因此, 解理后的 $\mathrm{Bi}-2212$ 单晶的表面即是 $\mathrm{Bi}-\mathrm{O}$ 面. $\mathrm{Bi}-\mathrm{O}$ 面内 $\mathrm{Bi}-\mathrm{Bi}$ 原子(或 $\mathrm{O}-\mathrm{O}$ 原子) 的距离 为 $3.8 \AA$.

本实验是用自行研制的低温 STM 进行 的. 本 STM 采用弹簧减速系统作粗调, 压电 陶瓷管扫描器, STM 探头被封在铜密封罩内, 以 In 圈压封, 详细结构请见参考文献 [11]. STM 针尖是用 $\varnothing 0.25 \mathrm{~mm}$ 的 $\mathrm{Pt}-\mathrm{Ir}$ 丝䇂成.在 装备研究样品之前, 先用高定向石墨检验针尖 的质量. 当观察到清晰的 $\mathbf{C}$ 原子的象以后, 再 换上新解理的 $\mathrm{Bi}-2212$ 单晶样品. 密封后, 用 扩散原将 STM 探头系统抽真空, 直到真空表 指示 $4 \times 10^{-6} \mathrm{~Pa}$. 探头被插人充满液 氮的不锈钢杜瓦瓶中, 待冷却 2-3h 后, 温度稳定在 $77 \mathrm{~K}$,开始采集 STM 数据.

根据 $\mathrm{Bi}-2212$ 单晶能带计算的结 果 ${ }^{[2,13]}, \mathrm{Bi}-\mathrm{O}$ 面费米面上下 $500 \mathrm{mV}$ 左右的电子态密 度 (DOS) 主要是由 $\mathrm{Bi}$ 的 $6 p$ 电子贡献的. 因此,当偏压为 $V_{b}= \pm 500 \mathrm{mV}$ 左右时, STM 图象中的亮点代表 $\mathrm{Bi}$ 原子.

\section{2 结 果 与 讨 论}

图 2 给出了 $77 \mathrm{~K}$ 下 $\mathrm{Bi}-\mathrm{O}$ 面的原子分辨的 STM 图象, 尺寸为 $220 \AA$ (横向) $\times 260 \AA$ (纵向). 该图象是在样品偏压为 $-530 \mathrm{mV}$, 隧道电流为 $0.72 \mathrm{nA}$ 下测到的. 由图可见笔直 的 $\mathrm{Bi}$ 原子链由上而下斜着贯穿全图, 没有水平面内的正弦调制,也没有 $\mathrm{Bi}$ 子列的丢失或压下. 沿 $a$ 方向的调制结构既不明显也不连贯, 不同于参考文献 [2-6]的结果. 图中特别亮和与其 相邻的特暗的区域, 可能是晶面上存在着 $1 / 2$ 元胞 $(15.5 \AA)$ 的断层的结果, 另外一些白亮点是 噪音干扰的结果.

图 3(a)是 $54 \AA \times 66 \AA$ 尺度的原子分辨的 $\mathrm{Bi}$ 原子图象, 该数据只被作了平滑处理. 靠 近图象底部的横线是噪声干扰引起的. 图 3(b) 是图 3(a) 的謜三维画法. 这是迄今所见到的 


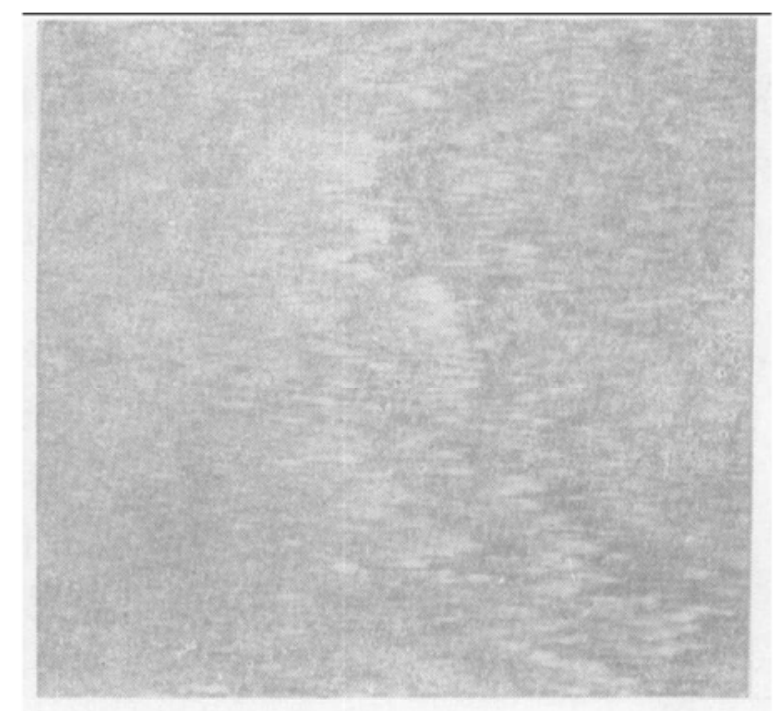

图2 77K 下 Bi-2212 单晶 Bi-O 面 STM 图象 $(220 \AA \times 260 \AA)$ 样品偏压为 $-530 \mathrm{mV}$, 随道电流为 $0.72 \mathrm{nA}$
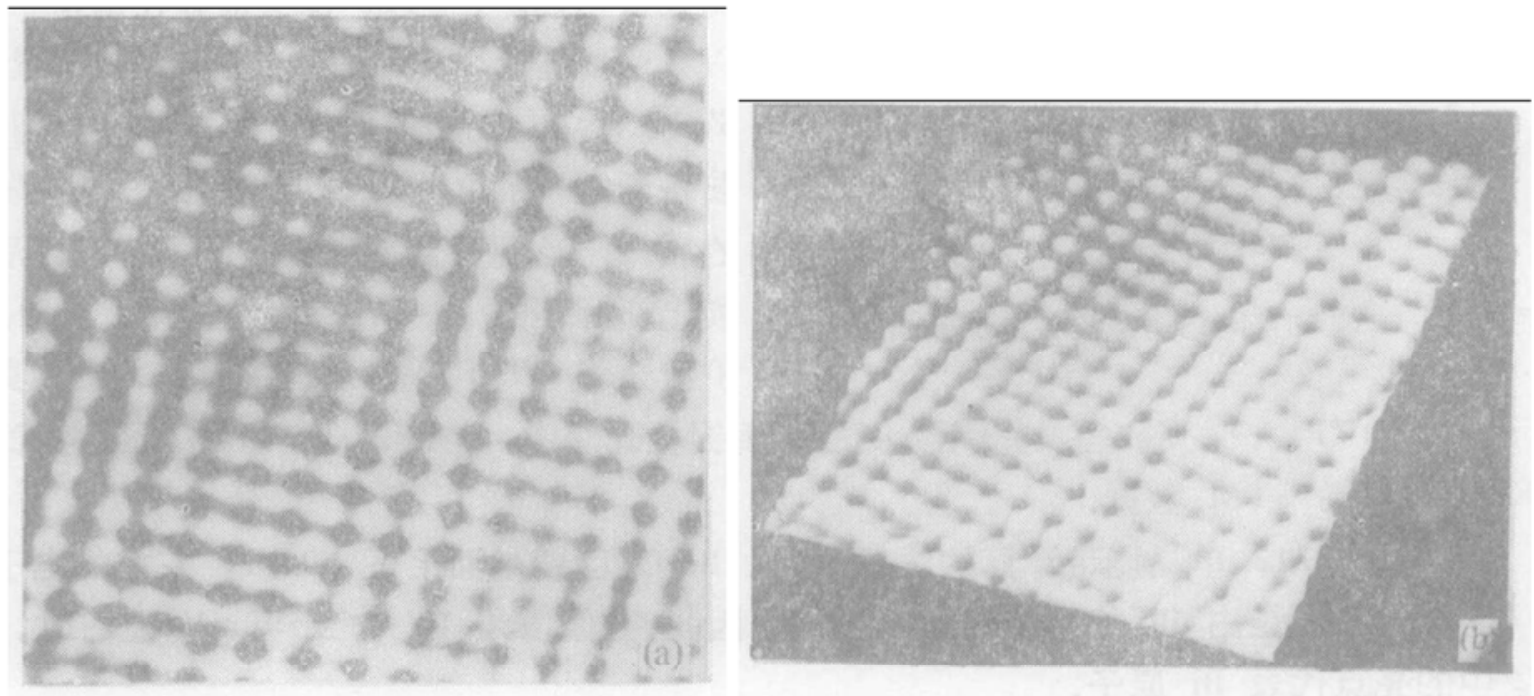

图 3

(a) $54 \AA \times 66 \AA \mathrm{STM}$ 图象偏压为 $-530 \mathrm{mV}$, 隧道电流 $0.72 \mathrm{nA}$. (b)(a) 的原三维图

最清晰的超导态的 $\mathrm{Bi}-2212$ 的 $\mathrm{Bi}-\mathrm{O}$ 面的 STM 图像. 我们测得 $\mathrm{Bi}-\mathrm{Bi}$ 原子间距为 $3.7 \pm$ $0.1 \AA$, 垂直于平面方向上 $\mathrm{Bi}$ 原子(亮点)和 $\mathrm{Bi}$ 原子间的缝隙(暗点)之间的起伏为 $0.4 \AA$ 左 右. 与图 2 一样,在此高分辨图像上, 观察不到水平方向的正弦形调制, $\mathrm{Bi}-\mathrm{Bi}$ 原子组成完整 的四方格子. 该图也没有显示出 $\mathrm{Bi}$ 原子列的失踪或被压到平面以下,没有连贯的一维超结构 调制. 但,由图中明暗祄度的不同可知, 在 $a b$ 面上下 ( $c$ 方向) Bi 原子位置的起伏是存在的.

\section{3 结 论}

利用自制的低温 STM 对 Bi-2212 单晶的 $\mathrm{Bi}-\mathrm{O}$ 面在 $77 \mathrm{~K}$ 下(超导态) 进行了实验研 
究, 获得了高分辨的 STM 图象. 与文献 [2-6]报道的不同, 在本实验所使用的偏压和隧道 电流下, 我们的原子分辨的 STM 图象没有显示出连贯的一维无公度结构调制, 在 $a b$ 面内 Bi 原子形成完整的四方格子,没有可辨认的正弦形调制, 也没观察到 $\mathrm{Bi}$ 原子列的失踪或被 压到下面去. 本实验对用 STM 观察到一维无公度调制结构的形成机制提出了新挑战. 本 实验表明一维无公度调制结构的 STM 观察是与样品偏压、样品与针尖之间的距离, 以及样 品表面的电子状态等密切相关的. Bi 原子链在 $a b$ 面内的正弦形调制可能是与样品有关的, 而并非 Bi-2212 单晶表面本征的特性.

进一步在 $4.2 \mathrm{~K}$ 下的实验和扫描隧道谱(STS)的研究正在进行中.

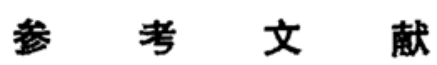

[1] Raveau, B., Michel, C., Studer, F. et al., Studies of High Temperature Swper-Condwctors, Vol. 9(ed. Narlikar, A.), Noav Science, New York, 1991, 81.

[2] Kirk, M.D., Nogami, J., Baski, A.A. et al., Science, 1988, 242:1673-1675.

[3] Shih, C. K., Feensta, R.M., Kirtley, J.R. et al., Phys. Rev. B, 1989,40:2682 - 2685.

[4] Shih, C.K., Feenstra. R.M., Chandrashekhar, G.V., Phys. Rev., B, 1991,43: $7913-7922$.

[5] Wu Xian-liang, Zhang Zhe, Wang, Yue-1i et al., Science, 1990,284:1211-1214.

[6] Zhang Zhe, Lieber, C.M., Phys. Rev., B, 1992,46:5845-5848.

[7] Samanta, S.B., Dutta, P.K., Awana, V.P.S. et al., Physica, C, 1991, 178:171-181.

[8] Wang Chen, Giambattista, B., Slough C.G., Phys. Rev., B, 1990, 42:8890-8906.

[9] Hasegawa, T., Nantoh, M., Kitazawa, K., Jpn.J. Appl. Phys., 1991, 30:L276-279.

[10] Liu Jin-xiang, Wan Ji-chun, Goldman, A.M., Phys. Rev. Lett., 1991, 67:2195-2198.

[11] 高凡、戴长泰、陈增波等,科学通报, 1993,38(13): 1186-1187.

[12] Hybertsen, M.S., Matthesis, L.F., Phys. Rev. Lett., 1988, 60: 1661-1664.

[13] Krakauer, H., Pickett, W.E., Phys. Rev. Lett., 1988, 60:1665-1667. 